\title{
Evidence that Climbing Fibers Control an Intrinsic Spike Generator in Cerebellar Purkinje Cells
}

\author{
Nadia L. Cerminara and John A. Rawson \\ Department of Physiology, Monash University, Clayton Victoria 3800, Australia
}

It is well established that the climbing fiber (CF) input to a cerebellar Purkinje cell (PC) can exert a controlling influence on the background simple spike (SS) activity of the cell, in that repetitive stimulation of CFs causes a decrease in SS activity, and removal or inactivation of CFs is followed by a rise in activity. In the present study, the effects of inactivation of CFs in the short term and longer term (hours) were investigated in anesthetized rats to determine how the CFs control the PC SS activity. Inactivation of the CF input to a PC was accomplished by either reversibly inactivating with lignocaine or by microlesioning the inferior olive. Consistent with previous findings, CF removal caused a transformation of the PC firing pattern, with SSs discharging more regularly and rising to an exceptionally high level. In cases in which CF activity resumed, SS rate declined to control levels within a few seconds. However, with sustained CF inactivation ( 30 min to $5 \mathrm{hr}$ ), SS activity continues to rise progressively and develops an oscillating firing pattern, consisting of alternating bursts of high-frequency discharge at up to $100-150 \mathrm{~Hz}$ followed by $10-20 \mathrm{sec}$ periods of electrical quiescence. No accompanying changes in the threshold for evoking SSs via the parallel fibers were seen to accompany the increases in tonic SS activity. We conclude that the increase in SS activity that follows CF inactivation could be caused by the removal of an inhibitory action that CFs exert on the intrinsic pacemaker present in PCs under normal conditions.

Key words: cerebellum; inferior olive; climbing fiber; Purkinje cells; pacemaker; rat

\section{Introduction}

Purkinje cells (PCs) are the sole output neurons of the cerebellar cortex, and they occupy a central position in cerebellar circuitry. They receive information via two distinct afferent systems: the mossy fiber-parallel fiber (MF-PF) pathway and the climbing fiber (CF) pathway. These two pathways each generate a distinct postsynaptic effect on the PC.

Activation of the MF-PF pathway generates single $\mathrm{Na}^{+}$dependent soma action potentials, or simple spikes (SSs), which can attain rates of $>200 \mathrm{~Hz}$ during motor performance (Thach, 1967). CF activation evokes a powerful and infrequent all-ornone complex spike (CS), consisting of a brief burst of ordinary action potentials and $\mathrm{Ca}^{2+}$-dependent dendritic spikes (Eccles et al., 1966a). In contrast to SSs, CS discharge occurs at very low rates, $\sim 1.5 \mathrm{~Hz}$ in quiescent animals (Armstrong and Rawson, 1979). Because SS frequency modulation appears to dominate the $\mathrm{PC}$ output during behavioral changes, the MF-PF system is thought to be important for the moment to moment operation of the cerebellum. However, despite their low firing rate, it is recognized that CFs are essential for normal cerebellar function, because CF loss produces deficits as severe as those after cerebellectomy (Llinas et al., 1975).

\footnotetext{
Received Aug. 28, 2003; revised March 6, 2004; accepted March 22, 2004.

This work was supported by the National Health and Medical Research Council of Australia. We are indebted to Prof. D. M. Armstrong for his comments on this manuscript.

Correspondence should be addressed to Dr. Nadia Cerminara, Department of Physiology, School of Medical Sciences, University of Bristol, University Walk, Bristol BS8 1TD, UK. E-mail: n.cerminara@bristol.ac.uk. DOI:10.1523/JNEUROSCI.4530-03.2004

Copyright $\odot 2004$ Society for Neuroscience $\quad$ 0270-6474/04/244510-08\$15.00/0
}

PCs are also known to display tonic SS firing in the absence of any overt stimuli. For many years it had been thought that the resting discharge of cerebellar PCs is produced by the sustained postsynaptic depolarization of the cells by ongoing transmission along the MF-PF pathway, but there has long been good reason to doubt this. For example, chronic deafferentation of the cerebellum does not abolish spontaneous SS activity (Eccles et al., 1967) and in agranular cerebella, PCs maintain high levels of ongoing SS discharge (Woodward et al., 1974; Siggins et al., 1976). In vitro studies have also shown the presence of endogenous ionic conductances that can generate spike activity in the PCs (Llinas and Sugimori, 1980a,b; Hounsgaard and Midtgaard, 1988; Raman and Bean, 1997, 1999).

It has been suggested that one possible role of the CFs is to exert a powerful tonic inhibitory effect on PC activity. This has been based on the observation that inactivation of the inferior olive (IO), the sole source of CFs, is paralleled by a marked increase in PC SS discharge (Colin et al., 1980; Montarolo et al., 1982; Demer et al., 1985; Savio and Tempia, 1985; Leonard and Simpson, 1986). Conversely, repetitive stimulation of CFs was found to cause a progressive reduction and finally a total cessation of spontaneous SS activity (Colin et al., 1980; Rawson and Tilokskulchai, 1981; Demer et al., 1985). Combined with evidence that PC SS activity is produced intrinsically, it is therefore proposed that a role of CFs is to exert control on the intrinsic spike generator. To test this hypothesis, we used an in vivo rat preparation to examine the effects of CF inactivation on single PCs positively identified by the presence of evoked CS discharges after low-strength electrical stimulation of the IO. CF inactivation achieved either by microlesion or by the application of a local 
anesthetic to the parent cell or axon in the IO enabled us to examine the effect in a precise manner.

\section{Materials and Methods}

Surgical and recording procedures. Experiments were performed on adult Long-Evans male rats that weighed between 300 and $650 \mathrm{gm}$. Animals were obtained from the Central Animal Breeding facility of Monash University. All surgical and experimental procedures were approved and performed under the guidelines set by the local Monash University Animal Ethics Committee and the National Health and Medical Research Council of Australia.

The animals were anesthetized with an initial dose of sodium pentobarbitone (Nembutal; Rhone Merieux, Pinkenba, Australia) $60 \mathrm{mg} / \mathrm{kg}$ delivered intraperitoneally. Anesthesia was maintained by the inhalation of oxygen containing $0.5-2 \%$ halothane (Rhone Merieux) via a cannula inserted in the trachea. A dose of $6 \mu \mathrm{g}$ atropine sulfate, given intraperitoneally (AstraZeneca Atropine; AstraZeneca, North Ryde, Australia) was given to ensure a clear air passage. Anesthesia was maintained at a level at which there was no flexor withdrawal reflex in response to vigorous pinching of the skin between the toes. The animal was placed in a stereotaxic head holder, and its rectal temperature was maintained at $37^{\circ} \mathrm{C}$ with a thermostatic blanket. A craniotomy was performed to expose the dorsal surface of the cerebellum and brainstem. The overlying dura of the cerebellum was carefully removed. During microelectrode recordings, exposed tissue was covered with a $2-3 \mathrm{~mm}$ layer of agarose gel $(1.5 \%$ in $0.9 \%$ saline; Sigma, St. Louis, MO) to prevent dehydration and to minimize brain pulsations.

CNQX experiments. Initial experiments were undertaken to confirm that PCs generate SSs intrinsically. A dual micropipette-microelectrode, constructed by adhering a tungsten in glass microelectrode (3-5 M $\Omega$ ) to a glass micropipette with cyanoacrylate adhesive, was inserted into the cerebellar cortex to permit single-unit extracellular recordings of the PCs. PCs were identified by their characteristic CS discharge. A glassinsulated tungsten microelectrode (tip exposure, $15-25 \mu \mathrm{m}$ ) was positioned in the molecular layer of the cerebellar cortex $0.5-1.0 \mathrm{~mm}$ away and in the long axis of the folium of the PC under study to stimulate its PFs using 0.1 msec duration cathodal pulses. PF activation of the PC was indicated by an evoked SS response, and thresholds were in the order of 1-5 $\mu \mathrm{A}$. The micropipette (tip diameter, 10-15 $\mu \mathrm{m}$ ) containing 6-cyano-7-nitroquinoxaline-2, 3-dione disodium salt (CNQX), a potent selective antagonist of the ionotropic glutamate AMPA-kainate receptor (Renard et al., 1995) was infused around the identified PC to examine its effect on intrinsic PC activity. Block of PF transmission at the PF-PC synapse was achieved by infusing $50 \mu \mathrm{M}$ CNQX (Tocris Cookson, Bristol, UK) with a Picospritzer (General Valve Corporation, Fairfield, NJ). During CNQX infusion, the PFs were continually stimulated, and CNQX was infused until PF stimulation no longer evoked an SS response, which typically took between $30 \mathrm{sec}$ and $1 \mathrm{~min}$. After the termination of CNQX infusion, PF stimuli of up to $40 \mu \mathrm{A}$ were tested every $3.5 \mathrm{sec}$ to ensure that all PF input was blocked. To make a reliable check on the effectiveness of the blockage of an evoked SS, the stimuli were applied at a fixed time after the occurrence of a spontaneous SS rather than in random relation to the background spikes. This eliminated the possibility that blockages of SS were caused by stimuli falling within the refractory period of the cell.

CF removal experiments. A dual micropipette-microelectrode was inserted stereotactically (Paxinos and Watson, 1986) into the IO through the dorsal surface of the medulla, via the gracile nucleus at an angle of $30-40^{\circ}$ and to a depth of $3-3.5 \mathrm{~mm}$ from the medullary surface. The micropipette contained 5 or $10 \%$ lignocaine hydrochloride $(1 \mathrm{mg} / \mathrm{ml}$; Xylocaine; AstraZeneca) in $0.9 \%$ saline. Lignocaine reversibly blocks voltage-gated $\mathrm{Na}^{+}$channels, thereby preventing action potential initiation and conduction (Martin and Ghez, 1999). The microelectrode was connected to a constant current stimulator, so that the output could be switched from short-duration pulses for CF stimulation to a direct current (DC) for producing microlesions. CF field potentials evoked on the cerebellar surface by IO stimulation were mapped with a lightly sprung platinum ball electrode. Fine positioning of the micropipette-microelectrode was achieved by mapping evoked CF potentials on the cerebellar cortical surface until a region was localized in which weak $(<100 \mu \mathrm{A})$ stimuli applied to the IO evoked CF field potentials. This was necessary to ensure that the microelectrode-micropipette was very close to the parent olive cell so that the input to the PC could be removed with a very small lesion or injection of lignocaine, keeping the loss of CFs as selective as possible and minimizing the risk of damaging sources of MFs around the IO.

Glass-coated tungsten microelectrodes (3-5 $\mathrm{M} \Omega$ ) were then used to record extracellularly from single identified PCs where weak olivary stimuli evoked large CF potentials on the cerebellar surface. The presence of a low-threshold CF input to individual PCs was checked by showing that the stimuli evoked all-or-nothing CS discharges. If a cell was activated with stimulus strengths $>100 \mu \mathrm{A}$, it was excluded from further study, because experience showed that it was necessary to make relatively large lesions to inactivate such cells.

Once it was established that the stimulating electrode was close to IO cells or axons, the CF providing an input to the identified PC was removed, either by passing $15 \mu \mathrm{A}$ of DC through the microelectrode tip or by pressure-ejecting nanoliter quantities of lignocaine around the parent cell or axon in the IO. The successful removal of the CF was indicated by the disappearance of spontaneous CSs. The short-term and long-term influence of CF inactivation was examined by recording the effects on PC background SS activity over periods respectively of minutes, up to $5 \mathrm{hr}$, during which the PC was constantly monitored for the continued absence of CSs.

Thresholds for PF activation of the PCs. PF stimulation (as described above) was also used to assess whether the changes in PC SS activity after CF removal were caused by a change in transmission at the PF-PC synapses. This was performed by monitoring the thresholds for evoking a SS via the PFs before and after the removal of CF inputs. Stimuli were applied at intervals of $3.5 \mathrm{sec}$, and the stimulus amplitude was adjusted to determine the minimal current for evoking a SS. PF activation of the PC was indicated by an evoked SS response, and threshold was defined as the minimum current required to elicit a SS response. Thresholds were measured for a control period of $5 \mathrm{~min}$ before CF inactivation and for up to 1 $\mathrm{hr}$ in some cells after CF inactivation.

Data acquisition and analysis. Signals from the microelectrode were first led into a unity gain headstage amplifier and then to a main amplifier with a gain of $1000 \times$ and a filter system that was set at a bandpass between $100 \mathrm{~Hz}$ and $10 \mathrm{kHz}$. To ensure that recordings were made from single cells, the recorded waveform was used to trigger a digital storage oscilloscope, and the entire signal was continuously displayed to check for uniformity of spike size and shape and the absence of activity within the refractory period of the cell. Action potentials from single neurons were converted to standard transistor to transistor logic (TTL) pulses with a time-amplitude window discriminator. A laboratory data acquisition system (1401 Processor and Spike2 software; Cambridge Electronic Design, Cambridge, UK) was used to digitize the TTL pulses and compile interspike interval histograms (ISI) and frequency graphs. A PowerLab (ADInstruments, New South Wales, Australia) data acquisition system sampling at $20 \mathrm{kHz}$ and using Scope and Chart software for Windows was used to record the timing of the stimulating pulses and raw spike train data.

To quantify the effects of CF removal on PC activity, the frequency of action potential discharge was measured before, and at various time points after CF removal. Where appropriate, quantitative data are expressed as mean \pm SEM. A one-way ANOVA was used to test for significance, and post hoc comparisons were made using the Dunnett's multiple comparison tests. Significance was set at the significance level $(p)$ indicated. Similarly, the frequency of PC action potential discharge before the infusion of CNQX was compared with that measured during CNQX infusion and after $1-5$ min of exposure to the drug. Coefficients of variation (CV) (SD ISI/mean ISI) were calculated to describe the regularity of PC discharge at rest and after CF inactivation (Goldberg and Fernández, 1971). A paired Student's $t$ test was used to test for significance $(p<0.05)$. The threshold for PF activation before the removal of the CF was compared with the average measured at various intervals after CF removal. A number of similar experiments were then averaged. An unpaired Student's $t$ test was used to test for significance $(p<0.05)$.

Histology. At the completion of experiments, a reference site was 
A.

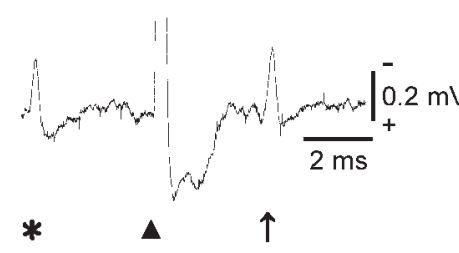

C.

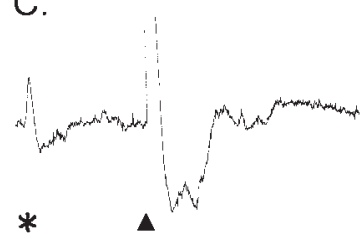

D.
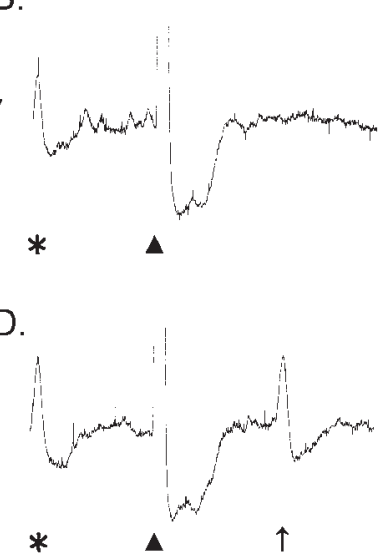

Figure 1. Effects of CNQX on PC responsiveness to PF stimulation before and after the infusion of $50 \mu \mathrm{m}$ CNQX. A, Arrow indicates an SS evoked with latency of $3.5 \mathrm{msec}$ by PF stimulation (triangle). B, C, Shortly after the addition of CNQX. Note the disappearance of the evoked SS.D, After the effect of CNQX has worn off, evoked SS activity returns. Spontaneously occurring SS indicated by asterisk. Each trace is an average of 10 records.

marked in the IO to verify the position of the electrodes. This was achieved by a microlesion, formed by passing $15 \mu \mathrm{A}$ of DC through the microelectrode tip for $30 \mathrm{sec}$. The animal was then killed with an overdose of sodium pentobarbitone. The brainstem and cerebellum were quickly removed and placed in a solution of $10 \%$ formalin with $20 \%$ sucrose until they had sunk. Sections were cut on a freezing microtome in the transverse plane at a thickness of $50 \mu \mathrm{m}$. Sections were mounted onto gelatinized slides, counterstained with neutral red, and dehydrated, cleared, and coverslipped. The sections were then examined under a light microscope to verify the position of the electrode in the IO.

\section{Results}

Confirming the intrinsic source of SSs: the effects of CNQX on tonic SS activity

Fast excitatory synaptic transmission at PF-PC synapses via the AMPA class of glutamate receptors (Renard et al., 1995) was abolished by infusing $50 \mu \mathrm{M}$ CNQX around the PC to establish that PCs generate SSs intrinsically. Typically, the time required to block evoked SS responses was from $30 \mathrm{sec}$ to $1 \mathrm{~min}$ after the commencement of infusion.

Application of CNQX totally blocked the SS responses to PF stimulation (Fig. $1 A-D$ ) but had little or no effect on the resting $\mathrm{SS}$ rate of most (12 of 18 ) of the PCs investigated (Fig. $2 A$ ). Some PCs (5/18) demonstrated a 20-30\% decrease in SS rate after infusion of CNQX (Fig. 2 B), whereas the remaining cell exhibited a $50 \%$ decrease. Overall, however, the SS resting firing rate of the population of PCs sampled remained unchanged (control = $46.04 \pm 6.2 \mathrm{~Hz}$; CNQX infusion $=44.19 \pm 6.0$; post $\mathrm{CNQX}=$ $40.65 \pm 6.26 \mathrm{~Hz} ; n=18 ; p>0.05$ ) (Fig. $2 D$ ). The persistence of much or all of the tonic SS activity after blockage of any ongoing $\mathrm{PF}$ input indicates that most of the background SS activity was generated intrinsically by the PCs.

It was notable that CNQX did not apparently block CF to PC transmission. For most of the PCs tested, CS discharge continued to occur at the same average frequency after CNQX application (10 of 18 ). Some PCs ( 5 of 18) demonstrated a $10-15 \%$ decrease in CS rate, whereas 3 of 18 displayed a $20-25 \%$ decrease. Overall, CNQX did alter CS resting rates (control $=1.00 \pm 0.10 \mathrm{~Hz}$; CNQX $=0.94 \pm 0.09 \mathrm{~Hz} ; n=18 ; p>0.05$ ). This suggests a differential sensitivity to this drug at the AMPA receptors of the $\mathrm{PF}$ and CF synapses. A likely explanation is that the receptors contain different subunits, as indicated by the finding that Joro
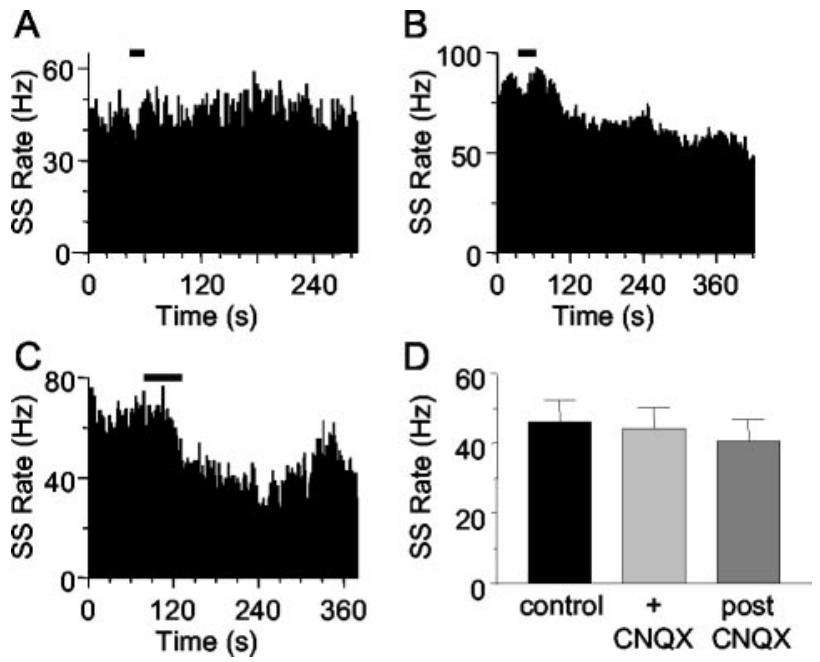

Figure 2. Effects of $50 \mu \mathrm{m} C \mathrm{NQX}$ on $S S$ rates. $A$, No change in $S S$ rate occurred for 12 of $18 \mathrm{PCS}$. $B$, Example $P C$ that displayed a $20-30 \%$ reduction in discharge rate ( 5 of 18 ). $C, A 50 \%$ decrease in SS rate (1 of 18). Bars in A-C represent period of CNQX infusion. Once PF stimulation could no longer evoke SSs, infusion was suspended. D, Comparison of SS rate on $18 \mathrm{PCs}$ before, during, and after CNQX infusion. Post-CNQX values were measured during a 1-5 min time window after infusion, during which no SSs could be evoked. Values are mean \pm SEM. Difference was not significant.

spider toxin blocks transmission through AMPA receptors at $\mathrm{PF}$ synapses but not at CF synapses (Ajima et al., 1991).

\section{Localization and identification of $\mathrm{CF}$ responses}

Surface recordings from the cerebellar cortex were made in each experiment before the removal of CFs to localize a region of cortex where CF activity was evoked by weak stimuli applied to the IO. Evoked responses were generally located in a narrow sagittal strip, corresponding to previous anatomical and electrophysiological observations that CFs branch and terminate in sagittal zones (Armstrong et al., 1973; Groenewegen and Voogd, 1977; Ekerot and Larson, 1982; Jörntell et al., 2000; Pardoe and Apps, 2002).

Responses produced in the cerebellar cortex by direct stimulation of CFs are known to produce large negative-positive or predominantly positive-going potentials with a constant latency and constant amplitude for a given stimulus strength (Armstrong and Harvey, 1968; Armstrong et al., 1973; Ekerot \& Larson, 1982). The latencies ranged between 3 and $4 \mathrm{msec}$ and remained constant from trial to trial. Figure $3 A$ shows a typical example of a CF field potential.

Extracellular recordings from individual PCs were then made in the area of cerebellar cortex displaying strong responses to $\mathrm{CF}$ activation. Identification of PCs was based on their orthodromic activation on stimulation of the IO to produce the characteristic all-or-nothing CS discharge. Figure $3 B$ shows an all-or-nothing CS response at threshold strength for CF activation. Recordings were made from 24 PCs where CSs could be generated by weak stimulation of the IO. Seventeen of the 24 cells (SS firing rate range, $4.84-78.63 \mathrm{~Hz}$ ) were inactivated over a period of minutes, whereas the remaining seven cells (SS firing rate range, 46.79$153.45 \mathrm{~Hz}$ ) were examined for periods of $>90 \mathrm{~min}$, and up to $5 \mathrm{hr}$ in one instance.

\section{Temporary removal of CFs}

The effects of a single intraolivary dose of lignocaine lasted from 5 to $10 \mathrm{~min}$, and sustained suppression of the CF input could be 
A.

B.

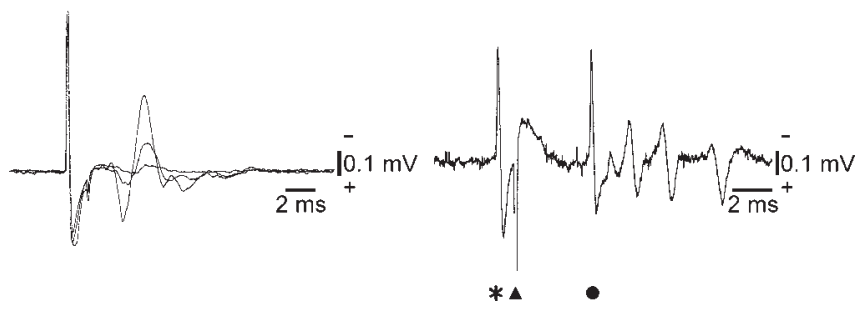

Figure 3. Identification of responses mediated by the stimulation of the CFs via the $10 . A$, Superimposed evoked responses recorded on the surface of the cerebellar cortex. CF field potential was recorded below, just above, and at $2 \times$ threshold (threshold $=12.5 \mu \mathrm{A}$ ). $B$, Example of an evoked CS generated by 10 stimulation. CS is marked by a dot at the initial response. A spontaneously occurring SS (asterisk) can be seen before the CS. Triangle refers to stimulus artifact.

achieved by repeat injection. After removal of the CF input, SS activity increased above its resting level within seconds of the disappearance of the spontaneous CSs (Fig. 4A). The SS discharge typically increased to a new steady level of discharge within 1-2 min of CF removal and continued to do so for the duration of CF removal (Fig. $4 B$ ). The rapid rise in SS firing was especially evident when extracellular spike train recordings of PC activity were examined before and after the removal of the $\mathrm{CF}$ input (Fig. 5Ai-Aii, Bi-Biii). This remarkable increase in SS discharge that accompanied the disappearance of the CSs was found to occur in all 17 PCs so that CF removal caused a significant increase in the SS activity of PCs from $27.59 \pm 5.34$ to $48.86 \pm$ $5.86 \mathrm{~Hz}(n=17 ; p<0.001)$ within $2 \mathrm{~min}$ of CF inactivation. SS discharge continued to rise for the duration of CF removal $(p<$ 0.001 ; time points $=5,10$, and $15 \mathrm{~min}$ ). These cells were sampled from different areas of cortex, and the response was similar among cells in different lobules or mediolateral positions in the cerebellum.

Once CF activity resumed, as indicated by the reappearance of spontaneous CSs (Figs. 4A, 5Ci), SS discharge declined to its initial control value. In contrast to the dramatic rise in SS firing rate that occurred after CF inactivation, the return to control levels after resumption of CS activity took somewhat longer, usually $2-3 \min (33.27 \pm 7.273 \mathrm{~Hz} ; n=10 ; p>0.05$; time point $=2$ min after CS return) (Figs. $4 \mathrm{~A}, 5 \mathrm{Ci}, \mathrm{Cii}$ ). The return of CS firing rate to its control level mirrored that of the SSs, in that some time was required before firing rates were equal to preinactivation levels. The form of the CS was also altered in the initial stages of its return. When compared with control (Fig. 5Ai, inset), CS form initially consisted of a large initial spike, with two or more wavelets or stunted spikes (Fig. 5Ci, inset). Two to $3 \mathrm{~min}$ after its first reappearance, CS form was restored to its previous profile of a relatively large initial spike and distinct, well formed secondary spikes (Fig. 5Cii, Ciii, inset).

\section{Longer term removal of CFs}

The effect of CF removal over longer periods was examined in an additional seven cells. Long-term inactivation was achieved by infusion of repeat doses of lignocaine or by an electrolytic microlesion in the IO. As with temporary inactivation, mean SS firing rate increased significantly from control values after CF removal ( control $=55.13 \pm 9.23 \mathrm{~Hz}$; inactivation $=84.10 \pm 7.36 \mathrm{~Hz}$; time point $=5 \mathrm{~min} ; p<0.05 ; n=7)$. PC SS activity continued to significantly increase $(p<0.001$; time points $=10-90 \mathrm{~min})$, attaining frequencies up to $100-150 \mathrm{~Hz}$ (Fig. 4C,D). However, after a period of 25-75 min, the discharge pattern of the cells
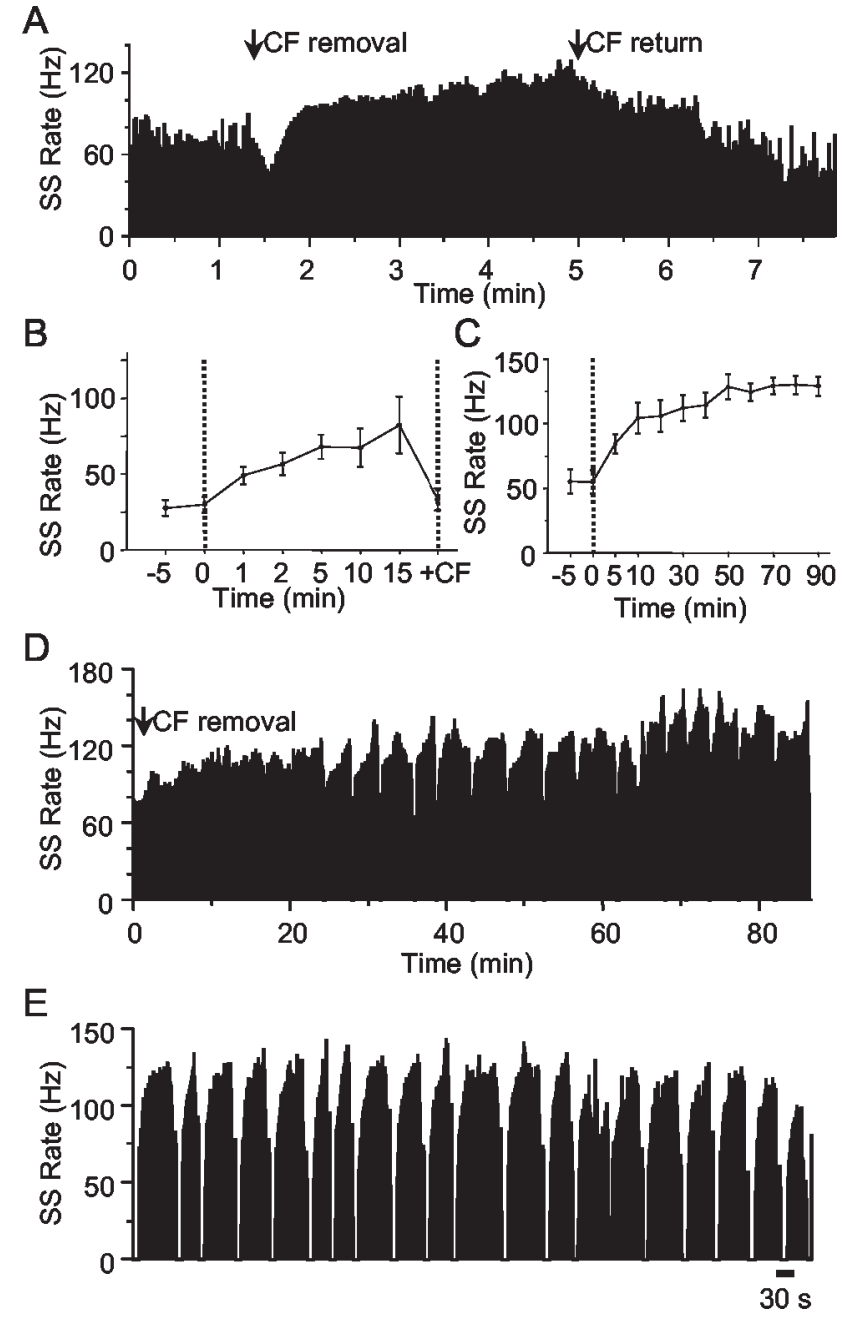

Figure 4. The effects of CF inactivation on SS rate. $A$, Short-term CF inactivation achieved with $5 \%$ lignocaine. $B$, SS discharge of 17 short-term inactivated cells at various time points after $C F$ removal. Each data point represents group mean $\pm S E M$. Dotted lines represent time of CF removal (time 0 ) and CF return ( + CF). C, SS rate of seven long-term inactivated $P C S$ at various time points after $C F$ removal. Each data point represents group mean \pm SEM. Dotted line represents time of CF removal. D, Long-term CF inactivation in which cell has developed oscillatory activity. CF removal was achieved with $10 \%$ lignocaine. E, PC that has been inactivated for $2 \mathrm{hr}$. Here the cell has entered its oscillatory mode, with activity fluctuating between periods of intense firing and quiescence. Bin widths, $1 \mathrm{sec}$.

became unstable, with cell firing arresting for $\sim 20 \mathrm{sec}$ before resuming once more. The cells then started to oscillate between periods of total silence and bursts of rising frequency discharge. After a period of $1.5-3 \mathrm{hr}$, the firing pattern of the PCs attained a strikingly regular period, exhibiting an oscillatory pattern of discharge of alternating periods of bursts of rapidly rising discharge with periods of quiescence lasting 10-20 sec (Fig. 4E). This oscillatory discharge was seen to persist for up to $5 \mathrm{hr}$ after CF removal. Furthermore, infusion of CNQX around CFinactivated PCs did not alter the rise in SS discharge (SS rate before CNQX $=82.72 \pm 0.52$; SS rate after CNQX $=92.13 \pm$ 2.78; $n=2$ ), nor did it alter the discharge when the cell had entered its oscillatory mode $(n=1)$.

Possible contribution of the CF pause

It is well established that a CS is often followed by a discernible pause in SS firing (Bloedel and Roberts 1971; Armstrong and Rawson, 1979). Thus, the removal of the CF input would remove 


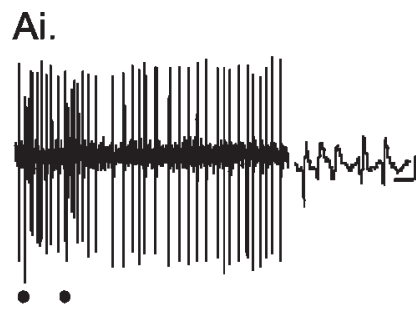

Aii.

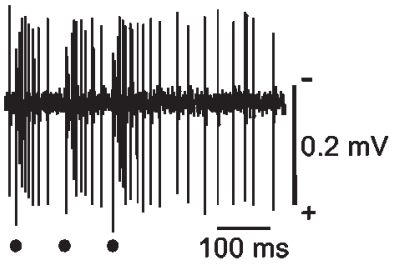

$\mathrm{Bi}$.

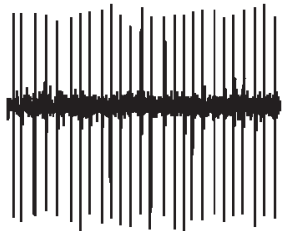

Bii.

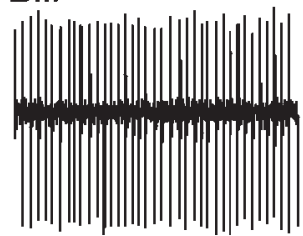

Biii.

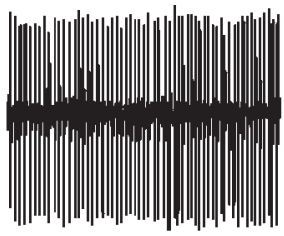

Ci.

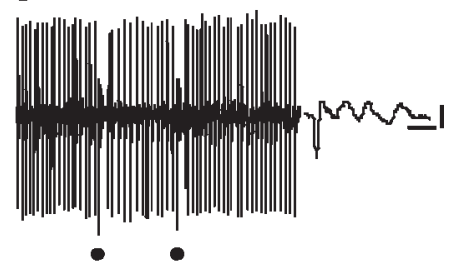

Cii.

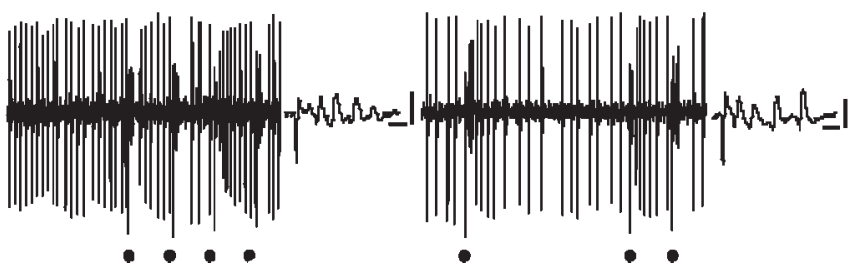

Figure 5. Recording of $\mathrm{PC}$ activity during CF inactivation with $5 \%$ lignocaine. Ai, Aii, Example of control spike trains, demonstrating the occurrence of both SSs and CSs. Bi-Biii, PC activity 1, 2 , and 3 min after its $C F$ has been removed. Note the disappearance of the $C S$ and the increased firing rate of the SSs. Ci-Ciii, Records of PC activity after CF inactivation, at 1, 2, and 3 min after the first reappearance of the CS. The SS rate progressively decreases with increasing CS discharge. The dots indicate the initial spike of the CS. Insets beside Ai, Ci-Ciii display the CS waveform on an expanded time scale. Calibration: $2 \mathrm{msec}, 0.1 \mathrm{mV}$.

a period of inhibition of SS activity with a possible contribution to the increase in SS firing seen after CF inactivation. Distinct post-CF pauses (i.e., the interval between the CS and the next SS) in the present PCs were brief $(29.6 \pm 2.3 \mathrm{msec} ; n=24)$ (Fig. 5). Given these brief pauses and the rate of CF discharge of $\sim 1 \mathrm{~Hz}$, removal of these pauses would amount to the approximate loss of a $30 \mathrm{msec}$ period of inhibition per second and may possibly contribute to the addition of $\sim 1$ SS per second. However, the removal of post-CF pauses would be a once only effect and cannot account for the sustained rise in SS seen after CF inactivation.

\section{ISI of CF inactivated PCs}

The firing patterns of PCs were irregular under normal control conditions, a fact reflected in the ISI histograms (Fig. 6 Ai, Bi). The $\mathrm{CV}$ of the ISI of short and long-term control PCs was $1.08 \pm 0.20$ (range $0.34-3.58 ; n=17$ ) and $0.75 \pm 0.14$ (range $0.37-1.44 ; n=$ $7)$, respectively. When the CF input to the cells was removed, the firing patterns of both short and long-term inactivated cells became strikingly regular as demonstrated by the ISI histogram shift to a more narrow distribution (Fig. 6 Aii,Bii). This was also

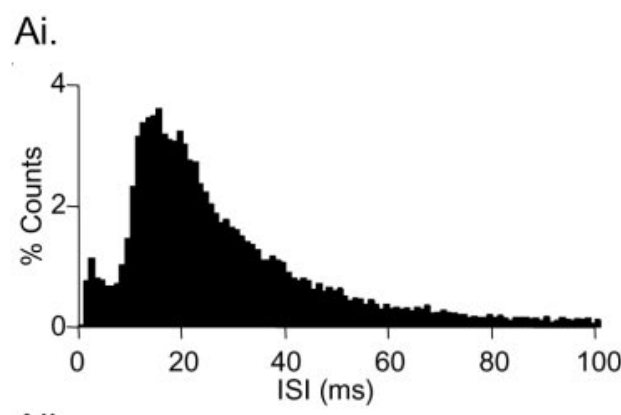

Aii.

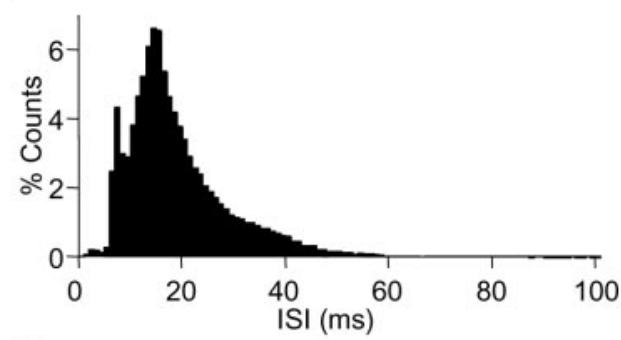

Bi.

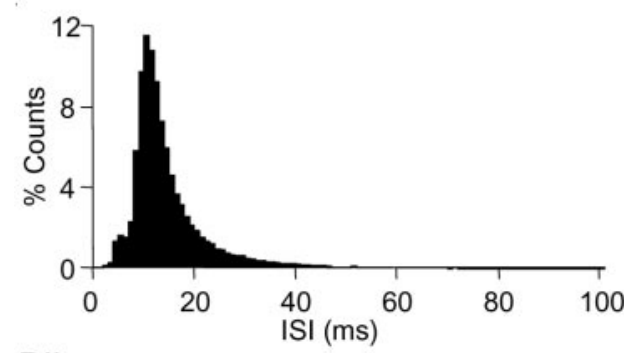

Bii.

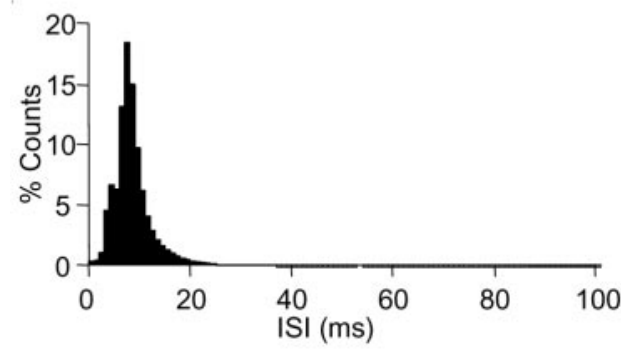

Figure 6. ISI histograms of short- and long-term inactivated PCs. CVs under the various conditions are given. Ai, Aii, ISI histograms of all short-term inactivated PCS ( $n=17)$. Ai, ISI histogram of SS activity before CF inactivation. Aii, When the CF was removed, the firing pattern of the $\mathrm{PC}$ became more regular, reflected in the narrow symmetric distribution of the ISI. Bi, Bii, ISI histograms of all long-term inactivated PCs $(n=7)$. Bi, Pre-CF removal; Bii, post-CF removal. Note the shift in ISI distribution after CF removal. The CVs were significantly decreased for both short- and long-term inactivated cells after CF removal.

reflected in the $\mathrm{CV}$, which was significantly reduced in both short $(0.46 \pm 0.08 ; p<0.05 ; n=17)$ and long-term inactivated cells $(0.43 \pm 0.06 ; p<0.05 ; n=7)$.

\section{Correlation of SS increase with control SS discharge}

To determine whether the change in SS rate after CF removal was related to the level of background SS activity, the maximal change in SS rate after CF removal was plotted against the control SS rate for all 24 cells (Fig. 7). No significant correlation was found between the increase in SS discharge rate and its control level of activity (coefficient of determination; $r^{2}=0.0055 ; p>0.05$ ). 


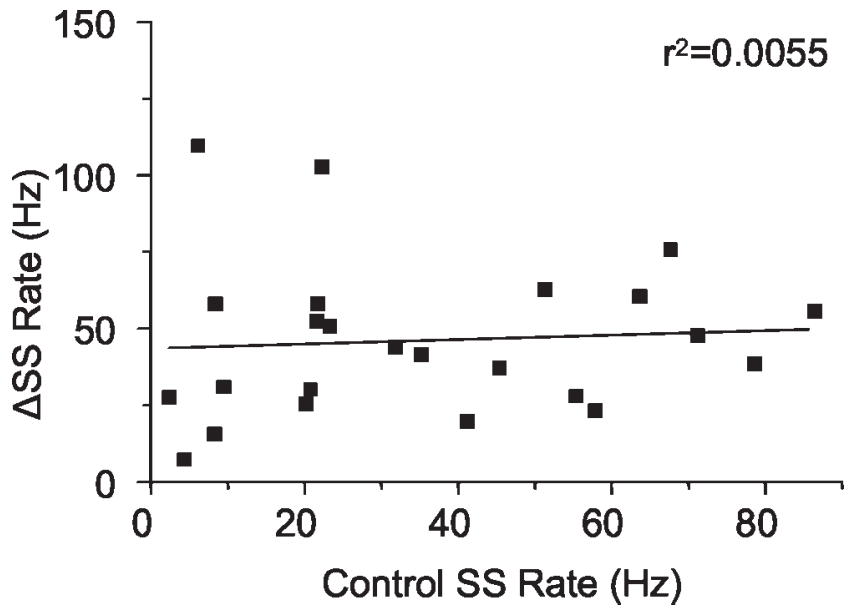

Figure 7. Correlation demonstrating the relationship of the change in $\mathrm{SS}$ rate after $\mathrm{CF}$ inactivation and the background discharge rate of the same cell for all $24 \mathrm{PCs}$ studied. Correlation coefficient $\left(r^{2}\right)=0.0055$.

\section{Restoration of long-term inactivated PCs}

Although lesioning a CF resulted in the removal of spontaneous CS activity, full degeneration of the CF requires some time, and therefore it is possible to stimulate $\mathrm{CF}$ axons for up to $18 \mathrm{hr}$ before the first sign of terminal degeneration (Desclin, 1976; Colin et al., 1980; Llinas and Sugimori, 1980a). As a result, in one experiment in which the CF input to a PC was removed electrolytically with a $\mathrm{DC}$ lesion, it was possible to reactivate the $\mathrm{CF}$ after a long period of inactivation.

Consistent with all PCs previously studied, SS activity quickly increased from a control discharge rate of $70.23 \pm 4.4 \mathrm{~Hz}$ to 130 $\mathrm{Hz}$ over a period of $40 \mathrm{~min}$, and then entered an oscillating mode, cycling between periods of discharge cessation and bursts of rising discharge at rates up to $150 \mathrm{~Hz}$ (Fig. 8A). After an inactivation period of $\sim 2 \mathrm{hr}, \mathrm{CF}$ activity was restored by electrical stimulation of their axons in the IO. A CF stimulation rate of only $1 \mathrm{~Hz}$ resulted in a return of SS firing rate to its preinactivation level (Fig. $8 A, B$ ). When the ISI histograms for this cell were examined, the distribution for normal control conditions and CF inactivation resembled that of all other PCs, shifting from a histogram with long-tailed appearance, to a narrower symmetrical distribution of ISIs during CF removal (Fig. 8Ci,Cii). After CF input had been restored, causing a decrease in SS firing frequency to that of preinactivation levels, the resulting ISI histogram also returned to a distribution matching that of control conditions (Fig. 8Ciii). This demonstrated that spontaneous SS activity was very dependent on CF activity and that even after a relatively long period of CF removal, resumed CF activity was capable of restoring PC SS activity to an apparently normal pattern.

\section{$\mathrm{PF}$ thresholds before and after $\mathrm{CF}$ inactivation}

The threshold for evoking a SS in the PC by direct stimulation of the PFs was measured before and after the removal of the CF input. This was done to assess whether the increase in SS activity after CF removal was caused by alterations in transmission at the PF-PC synapse. No corresponding changes in the threshold for evoking a SS via the PFs were seen to occur during the very marked and progressive elevations in SS rate that accompanied the loss of CF input (Fig. 9) $(p<0.05 ; n=11)$. Therefore, the significant changes in SS firing after CF removal occurred without an accompanying change in PF-PC transmission.
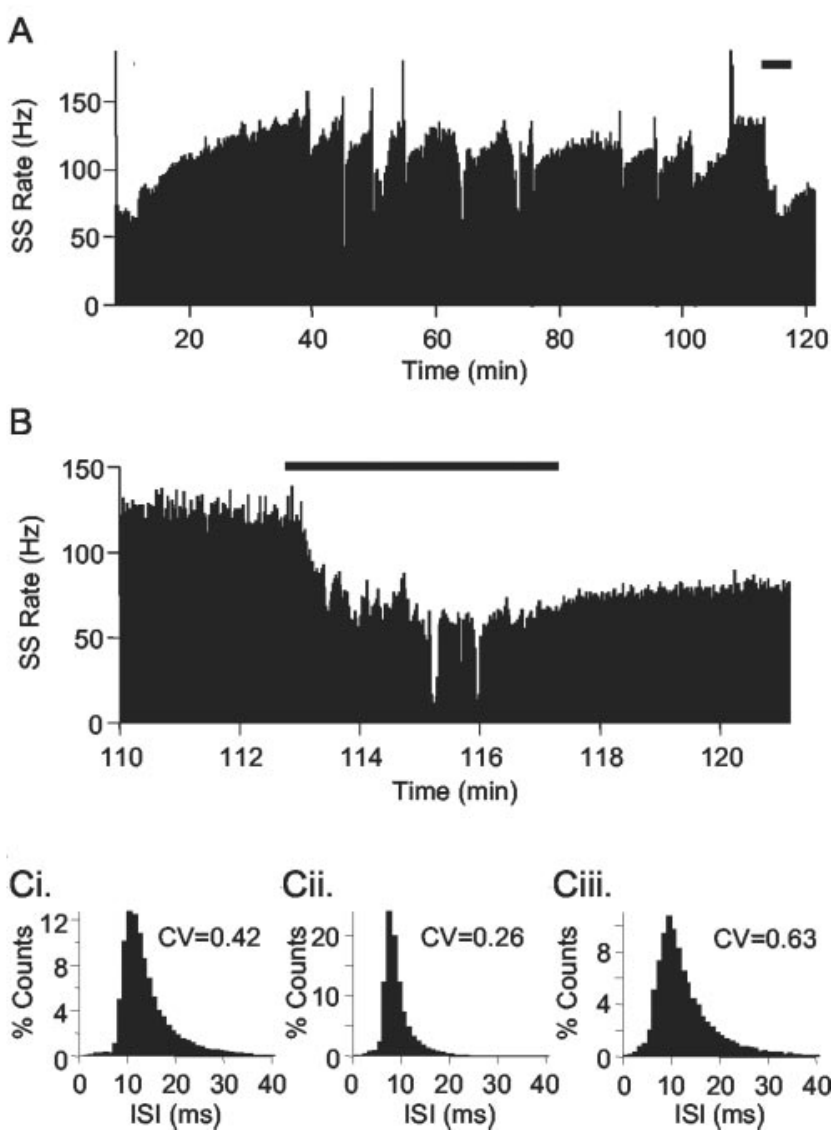

Figure 8. A, Effect of long-term CF inactivation on SS activity, followed by a period of CF stimulation. CF inactivation was achieved with a DC lesion. After a $2 \mathrm{hr}$ inactivation period, the CF was stimulated electrically at $1 \mathrm{~Hz}$. The period of stimulation is indicated by the length of the bar. Stimulation of the CF resulted in a return of SS activity toward its preinactivated level. $B$, Expanded time base of the CF stimulation period. C, ISI histogram of a PC whose CF has been removed, then stimulated after $2 \mathrm{hr}$ inactivation period. Ci, ISI of the control period. Cii, ISI of period of inactivation. Ciii, ISI after CF stimulation restored SS firing frequency to control levels.

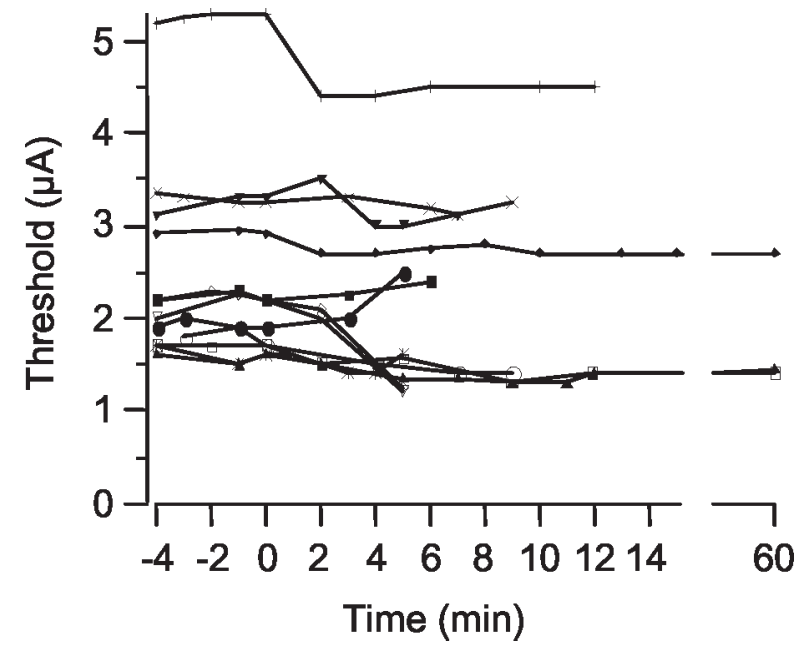

Figure 9. Plot demonstrating the threshold for evoking a SS by direct stimulation of the PFs, before and after the removal of the CF input. Time point 0 refers to the time of $C F$ removal.

\section{Discussion}

Spontaneous SS activity in vivo has previously been attributed to the continuous secretion of excitatory transmitter from PF synapses (Eccles et al., 1967). The present study has demonstrated 
that much or all of the background SS activity of PCs in vivo is generated intrinsically, as in the absence of excitatory PF inputs, PCs continued to display spontaneous SS activity. This finding is consistent with earlier studies that demonstrated that Purkinje cell SS discharge is preserved when synaptic transmission is blocked (Gähwiler, 1975; Häusser and Clark, 1997). The persistent firing of SSs that remains in the absence of functional PF synapses suggests that tonic SS activity is an inherent property of the PC.

The possibility that CFs can modulate the intrinsic spike generator was investigated by examining the effects of CF removal. Our observation of an increase in SS activity after the removal of its CF input is consistent with previous studies (Colin et al., 1980; Montarolo et al., 1982; Demer et al., 1985; Savio and Tempia, 1985; Leonard and Simpson, 1986). All these studies reported a salient increase in SS activity that accompanied the abolition of CS discharge. However, an observation not described in these previous studies was the prolonged rise in SS activity and subsequent oscillatory bursting pattern of SS discharge seen here after prolonged CF inactivation. Previous studies monitored the effect of CF inactivation over a period of minutes, and it is likely that this is the reason that this phenomenon remained previously undetected.

\section{Ionic conductances involved in SS control: a proposed model}

Previous models have proposed that the intrinsic SS activity of the PCs is generated primarily by a noninactivating or persistent $\mathrm{Na}^{+}$current, which is counterbalanced to a degree by a noninactivating $\mathrm{K}^{+}$current (Llinas and Sugimori, 1980a; Hounsgaard and Midtgaard, 1988; Raman and Bean, 1997, 1999). Although the model proposed here is similar in many regards to these previous models, it incorporates the new feature, namely that CFs exert control over the intrinsic spike-generating mechanism. The reason for focusing on these channels is that Llinas and Sugimori (1980b) showed that the blockade of $\mathrm{K}^{+}$channels in $\mathrm{Ca}^{2+}$-free medium caused the PC to depolarize dramatically to the point at which the spike-generating mechanism was inactivated. This suggests that the noninactivating $\mathrm{Na}^{+}$current is capable of powerfully depolarizing the PC in the absence of an opposing $\mathrm{K}^{+}$current.

The model proposed here to account for the rise in SS discharge after CF inactivation is that a change in cytosolic $\mathrm{Ca}^{2+}$ occurs as a result of loss of $\mathrm{Ca}^{2+}$ entry through voltage-operated $\mathrm{Ca}^{2+}$ channels (VOCCs) associated with CF activation (Eilers et al., 1995). When $\mathrm{Ca}^{2+}$ enters the PC cytosol, it is sequestered into internal stores over a time course of $\sim 10-15 \mathrm{sec}$ (Tank et al., 1988). Given that CFs discharge at rate of $\sim 1 \mathrm{~Hz}$, there will be insufficient time to fully sequester $\mathrm{Ca}^{2+}$ from the cytosol between successive CF impulses. The result will be a somewhat elevated level of free $\mathrm{Ca}^{2+}$ in the $\mathrm{PC}$, and this level will depend on the ongoing frequency of the $\mathrm{CF}$ input. This cytosolic $\mathrm{Ca}^{2+}$ will activate $\mathrm{Ca}^{2+}$-dependent $\mathrm{K}^{+}$channels, with a consequent hyperpolarization of the PC. It is predicted that when CF activity ceases, the PC will be deprived of its regular injections of $\mathrm{Ca}^{2+}$ through the VOCCs, and cytosolic $\mathrm{Ca}^{2+}$ will fall to a very low level. Consequently, current flow through $\mathrm{Ca}^{2+}$-dependent $\mathrm{K}^{+}$ channels will cease, and the PC will depolarize to the level set by the balance between the noninactivating $\mathrm{Na}^{+}$and $\mathrm{K}^{+}$conductances. Thus, SS rate should rise to the level determined by this depolarization within seconds after CF inactivation as $\mathrm{Ca}^{2+}$ is removed from the cytosol. This proposed mechanism is clearly consistent with the elevation in SS activity that occurred within seconds after CF inactivation.

However, it was also apparent from the present study that a PC progressively depolarizes over many minutes after CF inactivation, as indicated by the progressive increase in SS firing rate.
To account for this phenomenon, we propose that there is a corresponding decline in the ongoing outward $\mathrm{K}^{+}$current that normally counteracts the noninactivating $\mathrm{Na}^{+}$current. Thus, we envisage that the progressive depolarization of a PC over time after CF inactivation occurs as a consequence of the inward $\mathrm{Na}^{+}$ current becoming dominate.

This model can also explain the rhythmic firing characterized by alternating periods of high-frequency SS discharge and quiescence that occurred after long periods of CF inactivation. In this situation, PCs will become progressively depolarized (with a corresponding rise in SS rate) until the depolarization is sufficient to activate VOCCs, resulting in an influx of $\mathrm{Ca}^{2+}$ into the PC. The $\mathrm{Ca}^{2+}$ activates $\mathrm{Ca}^{2+}$-dependent $\mathrm{K}^{+}$channels that generate a hyperpolarization that inhibits $\mathrm{PC}$ firing. The $\mathrm{Ca}^{2+}$ is subsequently sequestered, and the hyperpolarizing current declines. The $\mathrm{Na}^{+}$ current then proceeds to depolarize the PC with a corresponding rapid rise in SS discharge, until the depolarization is again sufficient to open VOCCs and cause $\mathrm{Ca}^{2+}$ entry, and the cycle is repeated. It is thus envisaged that the oscillating behavior of the PCs results from a strong depolarization that is periodically interrupted by hyperpolarization generated by entry of $\mathrm{Ca}^{2+}$ and activation of $\mathrm{Ca}^{2+}$-dependent $\mathrm{K}^{+}$channels.

It may be noted that the oscillatory firing of the present PCs after removal of their CF input is remarkably similar to the spontaneous firing pattern of PCs in cerebellar slice preparations (Llinas and Sugimori, 1980a,b), in which the cells have been deprived of their CF input for some considerable time. The cell firing is characterized by periods of bursting and silence, with about the same period as described here. Moreover, $\mathrm{Ca}^{2+}$ imaging studies showed regular oscillations in cytosolic $\mathrm{Ca}^{2+}$ in PCs that displayed autorhythmic activity in slice preparations (Tank et al., 1988), and such oscillations were not seen in $\mathrm{Ca}^{2+}$-free media.

It is also notable that blockade of P-type VOCCs in cerebellar slices results in PCs firing in a bursting pattern (Edgerton and Reinhart, 2003), not unlike that seen here when CF input was blocked. Additionally, the blockade of small conductance (SK) $\mathrm{Ca}^{2+}$-dependent $\mathrm{K}^{+}$channels that are known to be activated by $\mathrm{Ca}^{2+}$ entry through P-type VOCCs (Edgerton and Reinhart, 2003) has also been shown to transform PC firing, dramatically increasing firing rates and causing cells to cycle between periods of depolarization and quiescence (Edgerton and Reinhart, 2003; Womack and Khodakhah, 2003).

\section{Role of cerebellar inhibitory interneurons}

A question that arises from the current findings is to what extent the effects of CF removal on SS activity occur as a result of loss of excitation of inhibitory interneurons by way of CF collaterals? This is especially pertinent because studies have shown that blockade of inhibitory synapses also causes PCs to fire more regularly and with an increased rate (Aubry et al., 1991; Häusser and Clark, 1997). The CNQX infusions applied here would almost certainly have blocked any ongoing excitatory transmission in a fairly wide area of cortex around the PC under study, including any ongoing excitatory transmission from granule cells and their PFs to the inhibitory interneurons. If this system were exerting a major influence on the SS activity seen in the present PCs, then blockage should have resulted in an increase in SS activity, because of disinhibition. However, as reported above, there was little change in SS activity after application of CNQX. Additionally, to account for the dramatic effects of CF removal on inhibitory interneurons, the synaptic contacts between CFs and interneurons would also need to exert long-lasting effects that would contribute significantly to the ongoing activity of the interneu- 
rons. However, it appears that the excitatory actions of CFs on molecular layer interneurons are weak or very phasic (Eccles et al., 1966b; Bloedel et al., 1972), and Golgi cells are predominantly inhibited by CF impulses (Schulman and Bloom, 1981).

\section{Functional Implications}

The results of the present study suggest that the CF input to a PC regulates the spike-generating system to maintain SS activity at a particular level. This would enable the CFs to determine a significant proportion of ongoing cerebellar cortical output and hence the tonic control exerted by the cerebellum on its target neurons in the brainstem and thalamus.

Control over intrinsic PC activity would also enable the CFs to determine the level of ongoing discharge against which PF driven SS responses will be superimposed, and this ongoing discharge could influence the signal-to-noise ratio of the evoked responses. Indeed, Leonard and Simpson (1986) have shown that SS modulations induced by visual or vestibular stimulation became superimposed on a much higher background of SS activity soon after inactivation of the CF input to the PC. It would clearly be worthwhile to investigate this superimposition phenomenon in more detail to determine how different rates of CF input and different levels of background SS activity actually influence the signal-to-noise ratio of an evoked PF response.

\section{References}

Ajima A, Hensch T, Kado RT, Ito M (1991) Differential blocking action of Joro spider toxin analog on parallel fiber and climbing fiber synapses in cerebellar Purkinje cells. Neurosci Res 12:281-286.

Armstrong DM, Harvey RJ (1968) Responses to a spino-olivo-cerebellar pathway in the cat. J Physiol (Lond) 194:147-168.

Armstrong DM, Rawson JA (1979) Activity patterns of cerebellar cortical neurones and climbing fibre afferents in the awake cat. J Physiol (Lond) 289:425-448.

Armstrong DM, Harvey RJ, Schild RF (1973) Branching of inferior olivary axons to terminate in different folia, lobules or lobes of the cerebellum. Brain Res 54:365-371.

Aubry A, Batini C, Billard JM, Kao RT, Morain P (1991) Tetrodotoxin induced calcium spikes: in vitro and in vivo studies of normal and deafferented Purkinje cells. Exp Brain Res 84:297-302.

Bloedel JR, Roberts WJ (1971) Action of climbing fibers in cerebellar cortex of the cat. J Neurophysiol 34:17-31.

Bloedel JR, Gregory RS, Martin SH (1972) Action of interneurons and axon collaterals in cerebellar cortex of a primate. J Neurophysiol 35:847-863.

Colin F, Manil J, Desclin JC (1980) The olivocerebellar system. I. Delayed and slow inhibitory effects: an overlooked salient feature of cerebellar climbing fibers. Brain Res 187:3-27.

Demer JL, Echelman DA, Robinson DA (1985) Effects of electrical stimulation and reversible lesions of the olivocerebellar pathway on Purkinje cell activity in the flocculus of the cat. Brain Res 346:22-31.

Desclin JC (1976) Early terminal degeneration of cerebellar climbing fibers after destruction of the inferior olive in the rat. Synaptic relationships in the molecular layer. Anat Embryol 149:87-112.

Eccles JC, Llinas R, Sasaki K (1966a) The excitatory synaptic action of climbing fibres on the Purkinje cells of the cerebellum. J Physiol (Lond) 182:268-296.

Eccles JC, Llinas R, Sasaki K (1966b) The inhibitory interneurones with the cerebellar cortex. Exp Brain Res 1:1-16.

Eccles JC, Ito M, Szentágothai J (1967) The cerebellum as a neuronal machine. Berlin: Springer.

Edgerton JR, Reinhart PH (2003) Distinct contributions of small and large conductance $\mathrm{Ca}^{2+}$-activated $\mathrm{K}+$ channels to rat Purkinje neuron function. J Physiol (Lond) 548:53-69.

Eilers J, Callewaert G, Armstrong C, Konnerth A (1995) Calcium signaling in a narrow somatic submembrane shell during synaptic activity in cerebellar Purkinje neurons. Proc Natl Acad Sci USA 92:10272-10276.

Ekerot CF, Larson B (1982) Branching of olivary axons to innervate pairs of sagittal zones in the cerebellar anterior lobe of the cat. Exp Brain Res 48:185-198.
Gähwiler BH (1975) The effects of GABA, picrotoxin and bicuculline on the spontaneous bioelectric activity of cultured cerebellar Purkinje cells. Brain Res 99:85-95.

Goldberg JM, Fernández C (1971) Physiology of peripheral neurons innervating semicircular canals of the squirrel monkey. III Variations among units in their discharge properties. J Neurophysiol 34:676-684.

Groenewegen HJ, Voogd J (1977) The parasagittal zonation within the olivocerebellar projection. I. Climbing fiber distribution in the vermis of cat cerebellum. J Comp Neurol 174:417-488.

Häusser M, Clark BA (1997) Tonic synaptic inhibition modulates neuronal output pattern and spatiotemporal synaptic integration. Neuron 19:665-678.

Hounsgaard J, Midtgaard J (1988) Intrinsic determinants of firing pattern in Purkinje cells of the turtle cerebellum in vitro. J Physiol (Lond) 402:731-749.

Jörntell H, Ekerot C, Garwicz M, Luo XL (2000) Functional organization of climbing fibre projection to the cerebellar anterior lobe of the rat. J Physiol (Lond) 522:297-309.

Leonard CS, Simpson JI (1986) Simple spike modulation of floccular Purkinje cells during the reversible blockade of their climbing fibre afferents. In: Adaptive processes in visual and oculomotor systems (Keller EL, Zee DS, eds), pp 429-434. Oxford: Pergamon.

Llinas R, Sugimori M (1980a) Electrophysiological properties of in vitro Purkinje cell somata in mammalian cerebellar slices. J Physiol (Lond) 305:171-195.

Llinas R, Sugimori M (1980b) Electrophysiological properties of in vitro Purkinje cell dendrites in mammalian cerebellar slices. J Physiol (Lond) 305:197-213.

Llinas R, Walton K, Hillman DE, Sotelo C (1975) Inferior olive: its role in motor learning. Science 190:1230-1231.

Martin JH, Ghez C (1999) Pharmacological inactivation in the analysis of the central control of movement. J Neurosci Methods 86:145-159.

Montarolo PG, Palestini M, Strata P (1982) The inhibitory effect of the olivocerebellar input on the cerebellar Purkinje cells in the rat. J Physiol (Lond) 332:187-202.

Pardoe J, Apps R (2002) Structure-function relations of two somatotopically corresponding regions of the rat cerebellar cortex: oliv-corticonuclear connections. The Cerebellum 1:165-184.

Paxinos G, Watson C (1986) The rat brain in stereotaxic coordinates. Sydney: Academic.

Raman IM, Bean BP (1997) Resurgent sodium current and action potential formation in dissociated cerebellar Purkinje neurons. J Neurosci 17:4517-4526.

Raman IM, Bean BP (1999) Ionic currents underlying spontaneous action potentials in isolated cerebellar Purkinje neurons. J Neurosci 19:1663-1674.

Rawson JA, Tilokskulchai K (1981) Suppression of simple spike discharges of cerebellar Purkinje cells by impulses in climbing fibre afferents. Neurosci Lett 25:125-130.

Renard A, Crépel F, Audinat E (1995) Evidence for two types of non-NMDA receptors in rat cerebellar Purkinje cells maintained in slice cultures. Neuropharmacology 34:335-346.

Savio T, Tempia F (1985) On the Purkinje cell activity increase induced by suppression of inferior olive activity. Exp Brain Res 57:456-463.

Schulman JA, Bloom FE (1981) Golgi cells of the cerebellum are inhibited by inferior olive activity. Brain Res 210:350-355.

Siggins GR, Henriksen SJ, Landis SC (1976) Electrophysiology of Purkinje neurons in the weaver mouse: iontophoresis of neurotransmitters and cyclic nucleotides, and stimulation of the nucleus locus coeruleus. Brain Res 114:53-69.

Tank DW, Sugimori M, Connor JA, Llinas RR (1988) Spatially resolved calcium dynamics of mammalian Purkinje cells in cerebellar slice. Science 242:773-777.

Thach Jr WT (1967) Somatosensory receptive fields of single units in cat cerebellar cortex. J Neurophysiol 30:675-696.

Womack MD, Khodakhah K (2003) Somatic and dendritic smallconductance calcium-activated potassium channels regulate the output of cerebellar Purkinje neurons. J Neurosci 23:2600-2607.

Woodward DJ, Hoffer BJ, Altman J (1974) Physiological and pharmacological properties of Purkinje cells in rat cerebellum degranulated by postnatal x-irradiation. J Neurobiol 5:283-304. 